\title{
The natural evolution of idiophatic epimacular membrane
}

\author{
Libor Hejseka, Alexandr Stepanov ${ }^{a}$, Alena Dohnalova ${ }^{b}$, Tereza Rehakova ${ }^{a}$, Nada Jiraskova ${ }^{a}$
}

\begin{abstract}
Background and Aims. Epiretinal membrane (ERM) refers to a semi-translucent tissue layer found on the inner surface of the retina especially in older people. Surgical treatment remains controversial, optimal timing for such treatment is difficult to determine and data on the natural evolution of this disorder are limited. In this study we evaluated the natural course of idiopathic epimacular membrane in 49 patients (53 eyes).

Materials and Methods. Retrospective evaluation of a group of 49 patients (53 eyes) with idiopathic epimacular membrane confirmed by biomicroscopy, photography and optical coherence tomography (OCT).

Results. Patient age ranged from 51-85 years (median 72). The average follow-up was 21.3 months $( \pm 14)$. Between the initial and final best corrected visual acuity (BCVA) there was no statistically significant difference although there was a significant tendency to decrease in BCVA (Spearman $P=0.05$ ) during the follow-up. Initial BCVA correlated with initial central retinal thickness (CRT), final CRT, final volume, and age. The final BCVA significantly correlated with all parameters measured.

Conclusion. BCVA during follow-up tended to decrease: difference of starting BCVA and final BCVA values depending on the time of monitoring is significant. This we attribute to a slow gradual progression of macular changes. But, initial and final BCVA measurements were not substantially different at the end. Thus, in the absence of any clear signs of ERM progression, we can safely postpone the decision whether to perform PPV.
\end{abstract}

Key words: epimacular membrane, natural course, surgery, PPV

Received: June 23, 2016; Accepted with revision: December 19, 2016; Available online: January 13, 2017 https://doi.org/10.5507/bp.2016.065

${ }^{a}$ Department of Ophthalmology, Faculty of Medicine in Hradec Kralove, Charles University in Prague and University Hospital Hradec Kralove, Czech Republic

${ }^{b}$ Institute of Physiology, $1{ }^{\text {st }}$ Faculty of Medicine, Charles University in Prague, Czech Republic

Corresponding author: Alexandr Stepanov, e-mail: stepanov.doctor@gmail.com

\section{INTRODUCTION}

The epiretinal membrane (ERM) appears as a transparent avascular film localized on the inner surface of the retina and resting on the internal limiting membrane (ILM). ERM can be idiopathic, where we assume a relationship of its development with an abnormality of the vitreoretinal divide and with detachment of the posterior vitreous membrane (PVD). ERM develops also secondarily as a reaction to other diseases of the eye: vascular occlusion, uveitis, trauma, intraocular surgery, rupture of the retina, etc.

Idiopathic ERM is a relatively common pathology in the elderly population. According to the Beaver Dam Eye Study and the Blue Mountains Eye Study the overall prevalence of ERM in these populations is $7-11.8 \%$, with a 5-year incidence of 5.3\% (ref. $\left.{ }^{1-3}\right)$.

In approximately $19-31 \%$, the affection is bilateral, and mostly asymmetric. ERM affects both sexes equally. The precondition for development of idiopathic ERM is related to PVD. In anomalous PVD, a residue of cortical vitreous humour may remain on the surface of the macula, or PVD induces dehiscence in ILM. The development of ERM is due to retinal glial cells (astrocytes and Muller cells) and RPE cells. However, myofibroblasts, fibroblasts, hyalocytes, and macrophages have also been identified on histological examination ${ }^{4}$.
There exist two principal types of epimacular proliferation: simple (type 2) and more complex (type 1). The ERM of type 2 outgrows the surface of the ILM and consist of a layer of glial cells producing collagen IV. The ERM of type 1 is more cellular, containing astrocytes, myofibroblasts, fibrocytes, macrophages and retinal pigment epithelial cells (RPE) together with glial cells ${ }^{5}$. The ERM of type 1 is more often due to the action of contractile cells, responsible for the development of traction complications.

Epiretinal proliferation is usually localized in the macula and very often it has a close relationship to the fovea. The membrane usually presents as a mild sheen or glint on the retinal surface. In the course of time, ERM becomes highly reflective and thickened. The ERM may then become more opaque, obscuring underlying retinal details. ERM also may induce a lamellar defect, called a pseudohole, which can mimic a full-thickness macular hole which is differentiated by OCT. Contracture of ERM produces distortion and wrinkling of the surface of the retina, and continuing traction may cause shallow detachment and/or cystic changes of the macula.

Traction forces on retinal vessels result in increased vascular tortuosity and straightening of the perimacular vessels. The process can lead to macular edema or cystoid macular edema.

To begin with, patients may be asymptomatic. They may then present with metamorphopsia, aniseikonia, 
Biomed Pap Med Fac Univ Palacky Olomouc Czech Repub. 2017 Mar; 161(1):100-106.

Table 1. Input and output parameters of the cohort under investigation.

\begin{tabular}{|c|c|c|c|c|c|c|c|c|c|c|}
\hline Eye No. & Age & $\begin{array}{c}\text { FU } \\
\text { (months) }\end{array}$ & $\begin{array}{c}\text { Baseline } \\
\text { BCVA }\end{array}$ & $\begin{array}{c}\text { Score } \\
\text { ETDRS }\end{array}$ & $\begin{array}{l}\text { Final } \\
\text { BCVA }\end{array}$ & $\begin{array}{c}\text { Score } \\
\text { ETDRS }\end{array}$ & $\begin{array}{c}\text { Baseline } \\
\text { CRT }\end{array}$ & $\begin{array}{l}\text { Final } \\
\text { CRT }\end{array}$ & $\begin{array}{l}\text { Baseline } \\
\text { volume }\end{array}$ & $\begin{array}{c}\text { Final } \\
\text { volume }\end{array}$ \\
\hline 1 & 65 & 13 & $20 / 20$ & 85 & $20 / 20$ & 85 & 397 & 359 & 10.4 & 10.3 \\
\hline 2 & 85 & 48 & $20 / 40$ & 70 & $20 / 200$ & 50 & 414 & 660 & 12 & 14.2 \\
\hline 3 & 79 & 12 & $20 / 125$ & 45 & $20 / 100$ & 50 & 401 & 398 & 11.8 & 11.9 \\
\hline 4 & 71 & 24 & $20 / 50$ & 65 & $20 / 50$ & 65 & 494 & 522 & 13.2 & 13.4 \\
\hline 5 & 72 & 18 & $20 / 50$ & 65 & $20 / 25$ & 80 & 341 & 319 & 12.9 & 10.2 \\
\hline 6 & 64 & 18 & $20 / 20$ & 85 & $20 / 20$ & 85 & 354 & 297 & 12.7 & 11.1 \\
\hline 7 & 72 & 26 & $20 / 25$ & 80 & $20 / 40$ & 70 & 330 & 343 & 11.9 & 12.8 \\
\hline 8 & 72 & 18 & $20 / 25$ & 80 & $20 / 20$ & 85 & 329 & 289 & 11 & 10.5 \\
\hline 9 & 80 & 30 & $20 / 40$ & 70 & $20 / 40$ & 70 & 355 & 280 & 10.2 & 10.1 \\
\hline 10 & 72 & 18 & $20 / 25$ & 80 & $20 / 25$ & 80 & 410 & 338 & 11.6 & 10.4 \\
\hline 11 & 77 & 27 & $20 / 20$ & 85 & $20 / 20$ & 85 & 334 & 332 & 10.4 & 10.5 \\
\hline 12 & 63 & 30 & $20 / 20$ & 85 & $20 / 25$ & 80 & 339 & 328 & 11.4 & 10.8 \\
\hline 13 & 76 & 72 & $20 / 25$ & 80 & $20 / 63$ & 60 & 463 & 503 & 13.3 & 13.9 \\
\hline 14 & 65 & 18 & $20 / 20$ & 85 & $20 / 25$ & 80 & 302 & 389 & 11.7 & 11.5 \\
\hline 15 & 79 & 7 & $20 / 25$ & 80 & $20 / 25$ & 80 & 417 & 381 & 11.5 & 11.2 \\
\hline 16 & 76 & 13 & $20 / 20$ & 85 & $20 / 25$ & 80 & 313 & 349 & 10.9 & 12.3 \\
\hline 17 & 68 & 8 & $20 / 20$ & 85 & $20 / 20$ & 85 & 310 & 309 & 12.2 & 12.4 \\
\hline 18 & 57 & 12 & $20 / 32$ & 75 & $20 / 25$ & 80 & 370 & 359 & 12.3 & 12.2 \\
\hline 19 & 72 & 12 & $20 / 25$ & 80 & $20 / 32$ & 75 & 447 & 556 & 13 & 15 \\
\hline 20 & 70 & 25 & $20 / 32$ & 75 & $20 / 32$ & 75 & 335 & 449 & 11.3 & 11.9 \\
\hline 21 & 65 & 36 & $20 / 25$ & 80 & $20 / 25$ & 80 & 429 & 275 & 11 & 10.3 \\
\hline 22 & 78 & 6 & $20 / 63$ & 60 & $20 / 63$ & 60 & 573 & 579 & 14.5 & 14.6 \\
\hline 23 & 68 & 16 & $20 / 25$ & 80 & $20 / 25$ & 80 & 255 & 254 & 10.6 & 10.6 \\
\hline 24 & 81 & 12 & $20 / 40$ & 70 & $20 / 32$ & 75 & 386 & 379 & 11.1 & 9.9 \\
\hline 25 & 84 & 27 & $20 / 40$ & 70 & $20 / 40$ & 70 & 357 & 348 & 10.7 & 10.8 \\
\hline 26 & 64 & 12 & $20 / 20$ & 85 & $20 / 20$ & 85 & 371 & 375 & 10.6 & 10.4 \\
\hline 27 & 74 & 6 & $20 / 25$ & 80 & $20 / 25$ & 80 & 310 & 302 & 11 & 10.8 \\
\hline 28 & 61 & 11 & $20 / 25$ & 80 & $20 / 32$ & 75 & 499 & 416 & 12.7 & 11.8 \\
\hline 29 & 64 & 24 & $20 / 32$ & 75 & $20 / 25$ & 80 & 340 & 279 & 10.3 & 9.8 \\
\hline 30 & 78 & 24 & $20 / 20$ & 85 & $20 / 20$ & 85 & 279 & 264 & 10.5 & 10.5 \\
\hline 31 & 78 & 24 & $20 / 16$ & 90 & $20 / 20$ & 85 & 339 & 351 & 10.8 & 11.1 \\
\hline 32 & 68 & 32 & $20 / 20$ & 85 & $20 / 20$ & 85 & 392 & 385 & 12.3 & 12 \\
\hline 33 & 73 & 6 & $20 / 63$ & 60 & $20 / 50$ & 65 & 546 & 546 & 11 & 11 \\
\hline 34 & 78 & 37 & $20 / 32$ & 75 & $20 / 32$ & 75 & 393 & 436 & 12.3 & 12.4 \\
\hline 35 & 79 & 22 & $20 / 20$ & 85 & $20 / 20$ & 85 & 306 & 303 & 11.6 & 11.3 \\
\hline 36 & 72 & 47 & $20 / 20$ & 85 & $20 / 20$ & 85 & 351 & 401 & 11.6 & 12.4 \\
\hline 37 & 72 & 47 & $20 / 20$ & 85 & $20 / 20$ & 85 & 280 & 270 & 10.3 & 10.4 \\
\hline 38 & 59 & 24 & $20 / 20$ & 85 & $20 / 10$ & 100 & 306 & 280 & 11.9 & 11.5 \\
\hline 39 & 57 & 18 & $20 / 80$ & 55 & $20 / 40$ & 70 & 355 & 372 & 10.3 & 11.1 \\
\hline 40 & 57 & 18 & $20 / 50$ & 65 & $20 / 63$ & 60 & 375 & 405 & 9.4 & 9.8 \\
\hline 41 & 73 & 7 & $20 / 32$ & 75 & $20 / 25$ & 80 & 579 & 561 & 13.6 & 13.6 \\
\hline 42 & 83 & 14 & $20 / 80$ & 55 & $20 / 63$ & 60 & 333 & 330 & 11.4 & 11.5 \\
\hline 43 & 61 & 3 & $20 / 25$ & 80 & $20 / 25$ & 80 & 318 & 320 & 11 & 11 \\
\hline 44 & 83 & 36 & $20 / 32$ & 75 & $20 / 32$ & 75 & 419 & 417 & 11.9 & 12.6 \\
\hline 45 & 79 & 3 & $20 / 40$ & 70 & $20 / 32$ & 75 & 819 & 625 & 12.6 & 13.3 \\
\hline 46 & 51 & 12 & $20 / 20$ & 85 & $20 / 20$ & 85 & 316 & 320 & 11.7 & 11.6 \\
\hline 47 & 69 & 18 & $20 / 25$ & 80 & $20 / 25$ & 80 & 314 & 320 & 10.1 & 10 \\
\hline 48 & 69 & 18 & $20 / 25$ & 80 & $20 / 25$ & 80 & 355 & 360 & 10.4 & 10.4 \\
\hline 49 & 70 & 8 & $20 / 25$ & 80 & $20 / 25$ & 80 & 257 & 250 & 10.5 & 10.5 \\
\hline 50 & 70 & 8 & $20 / 20$ & 85 & $20 / 20$ & 85 & 268 & 175 & 10.4 & 10.4 \\
\hline 51 & 78 & 43 & $20 / 25$ & 80 & $20 / 32$ & 75 & 457 & 440 & 12.2 & 11.8 \\
\hline 52 & 71 & 48 & $20 / 20$ & 85 & $20 / 25$ & 80 & 307 & 306 & 10.8 & 12.1 \\
\hline 53 & 79 & 12 & $20 / 25$ & 80 & $20 / 25$ & 80 & 317 & 353 & 10.5 & 11.6 \\
\hline
\end{tabular}


Table 2. Preoperative and postoperative parameters of 53 eyes.

\begin{tabular}{lcccccccc}
\hline & $\begin{array}{c}\text { Age } \\
(\mathrm{n}=49)\end{array}$ & $\begin{array}{c}\text { Follow-up } \\
\text { period }(\mathrm{M})\end{array}$ & $\begin{array}{c}\text { Input } \\
\text { CRT }\end{array}$ & $\begin{array}{c}\text { Output } \\
\text { CRT }\end{array}$ & $\begin{array}{c}\text { Input volume } \\
\text { of the macula }\end{array}$ & $\begin{array}{c}\text { Output volume } \\
\text { of the macula }\end{array}$ & $\begin{array}{c}\text { Input } \\
\text { BCVA }\end{array}$ & $\begin{array}{c}\text { Output } \\
\text { BCVA }\end{array}$ \\
\hline Mean & 71.69 & 21.28 & 376.53 & 372.77 & 11.458 & 11.500 & 0.74962 & 0.75132 \\
Median & 72.00 & 18.00 & 354.00 & 351.00 & 11.400 & 11.200 & 0.80000 & 0.80000 \\
Standard deviation & 7.869 & 14.013 & 96.860 & 100.659 & 1.0458 & 1.2734 & 0.257271 & 0.296038 \\
Minimum & 51 & 3 & 255 & 175 & 9.4 & 9.8 & 0.160 & 0.100 \\
Maximum & 85 & 72 & 819 & 660 & 14.5 & 15.0 & 1.250 & 2.000 \\
\hline
\end{tabular}

M - month, BCVA - best corrected visual acuity, CRT - central retinal thickness

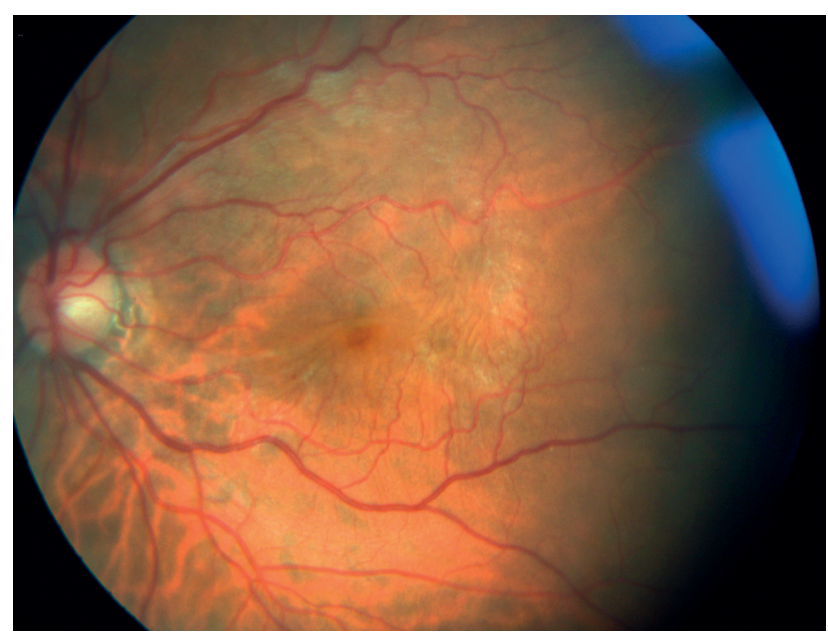

Fig. 1. High intensity of the epimacular membrane reflection and folding of the retinal internal limiting membrane.

monocular diplopia, and a variable loss of visual acuity ranging from 20/20 to 20/200 (ref. ${ }^{6}$ ). In most ERM cases, the symptoms are mild and surgical treatment is not necessary (indicated). The patient whose vision deteriorates to approximately 20/50 or worse, and/or has an intolerable metamorphopsia with better visual acuity may be a candidate for macular surgery by vitrectomy procedures (PPV). The ERM on the surface of the macula can be relatively easily removed by surgery. However, no recommendation has yet been issued concerning the timing of the surgery.

After ERM removal, 50\%-75\% of patients show some degree of improvement in vision, but return to normal vision is rare. The resultant visual functions in some cases are evaluated by the patient as unsatisfactory. Surgical solution may be complicated by the development of cataract, development of ruptures in the periphery of the retina, rhegmatogenous retinal detachment, endophthalmitis, loss of ganglial cells, etc. Most surgeons in the indication of PPV are guided by the initial visual acuity and their own clinical experience ${ }^{7}$.

\section{MATERIALS AND METHODS}

A prospective evaluation of a group of 49 patients (53 eyes) was performed. The best corrected visual acuity to distance discrimination (BCVA) was measured using the

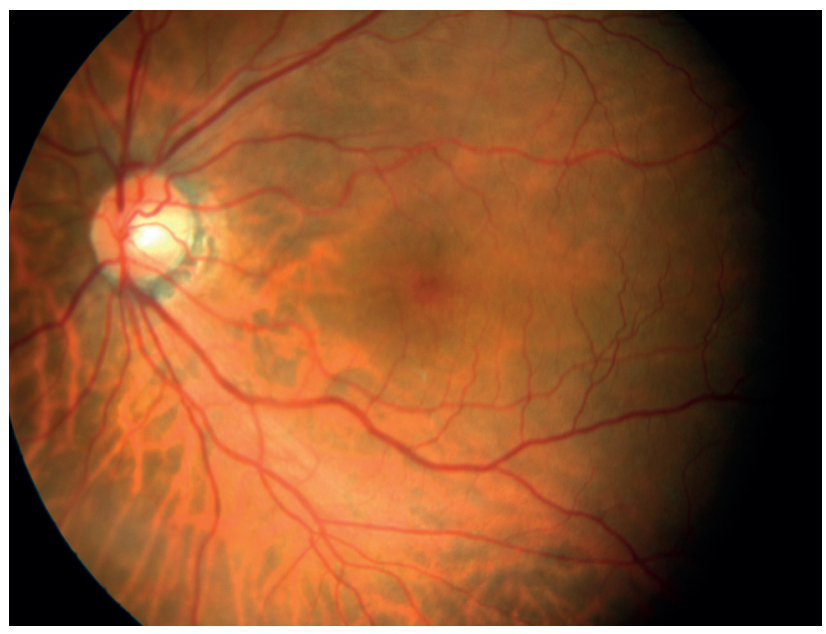

Fig. 2. Improvement of the state without treatment.

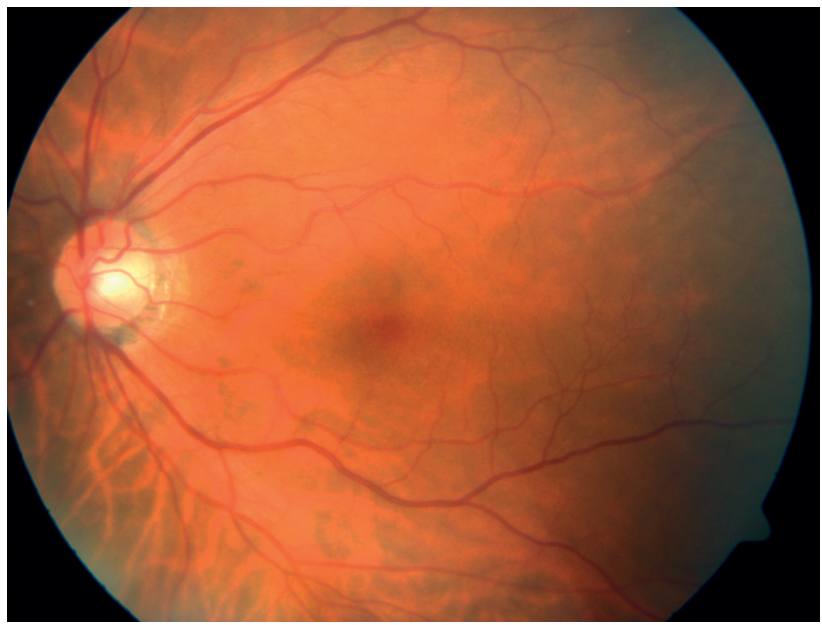

Fig. 3. Improvement of the state - resolution of epiretinal membrane.

ETDRS $\log$ MAR chart, central retinal thickness (CRT) and the volume of the central region were measured by means of Cirrus spectral OCT (Carl ZEISS). The finding on the eye background was evaluated biomicroscopically. The macular surface was also documented by colour photography (camera ZEISS, FF 450+). The parameters under investigation, namely the initial and final BCVA, CRT and the volume of the macula, were evaluated statistically by the nonparametric Wilcoxon test for 2 dependent se- 


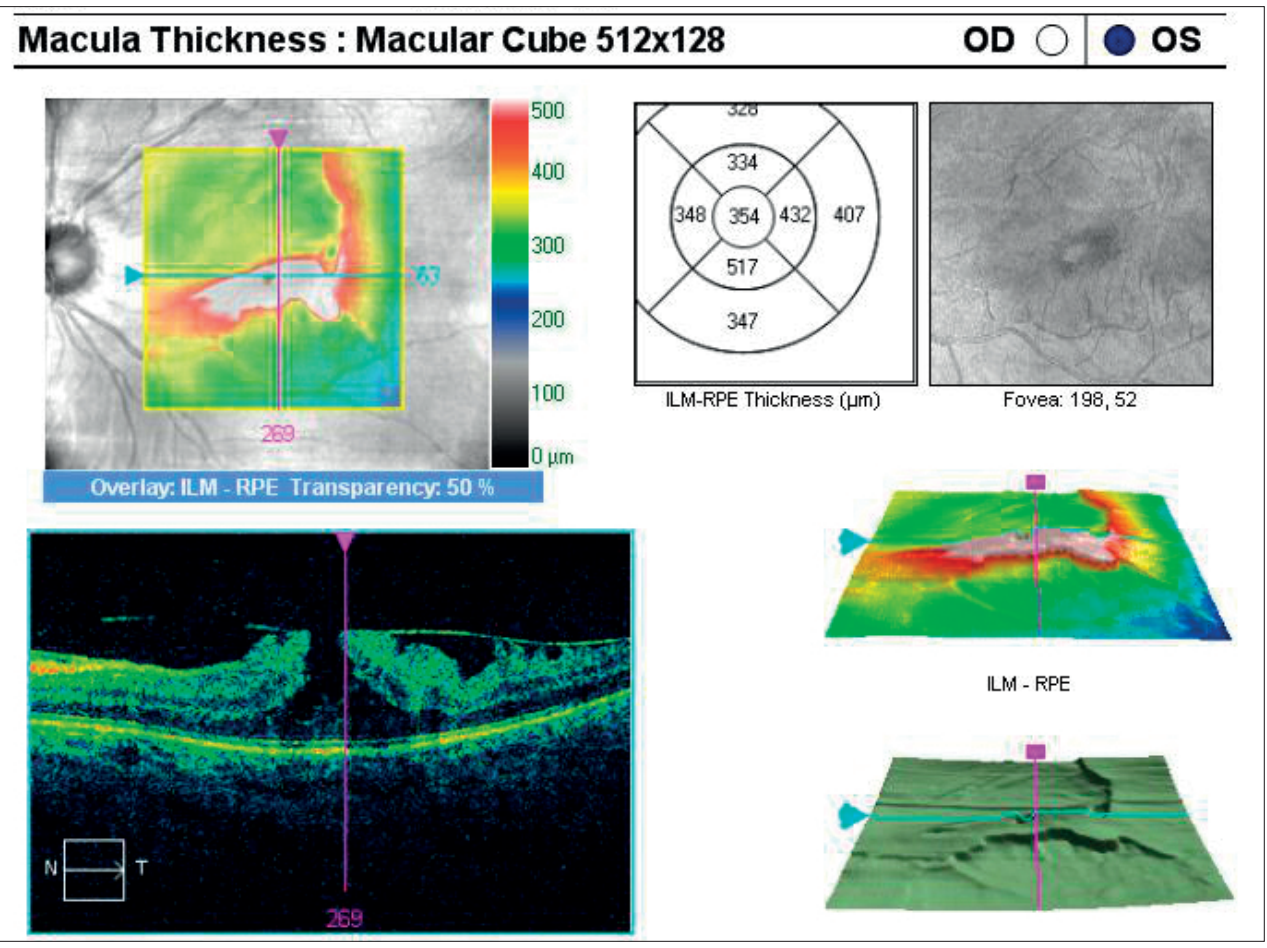

Fig. 4. Optical coherence tomography of the left eye: line of hyperreflectivity attached to the inner retinal surface, macular pseudohole and tangential vitreomacular traction.

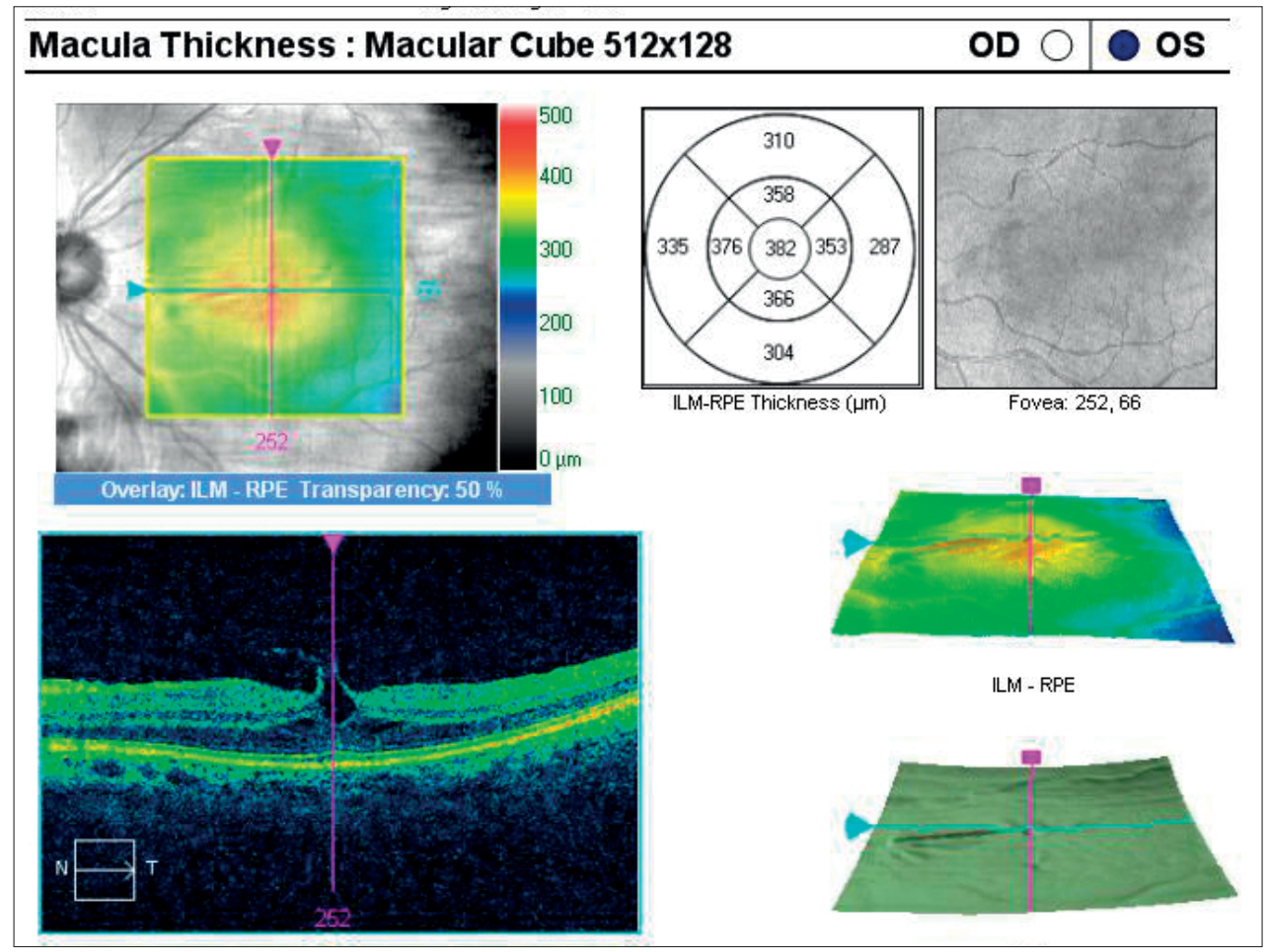

Fig. 5. Improvement of the state without treatment, focal vitreomacular traction.

lections. Statistical analysis was performed using SPSS 13.0 (Statistical Package for the Social Sciences, Chicago, USA). Spearman nonparametric correlation coefficient was employed to determine the mutual relationship of the quantities under investigation. The follow-up excluded patients who suffered from diabetic retinopathy or any condition after a vascular event of the retinal bed, who did not have peripheral degeneration or tear of the retina, whose posterior segment of the eye was not operated on (neither PPV nor cryosurgery), in whom there were no significant hypertonic changes in the vessels, and who had no pathological myopia. The most frequent reasons for not performing PPV was refusal by the patient because of satisfactory visual function and fear of surgery. 


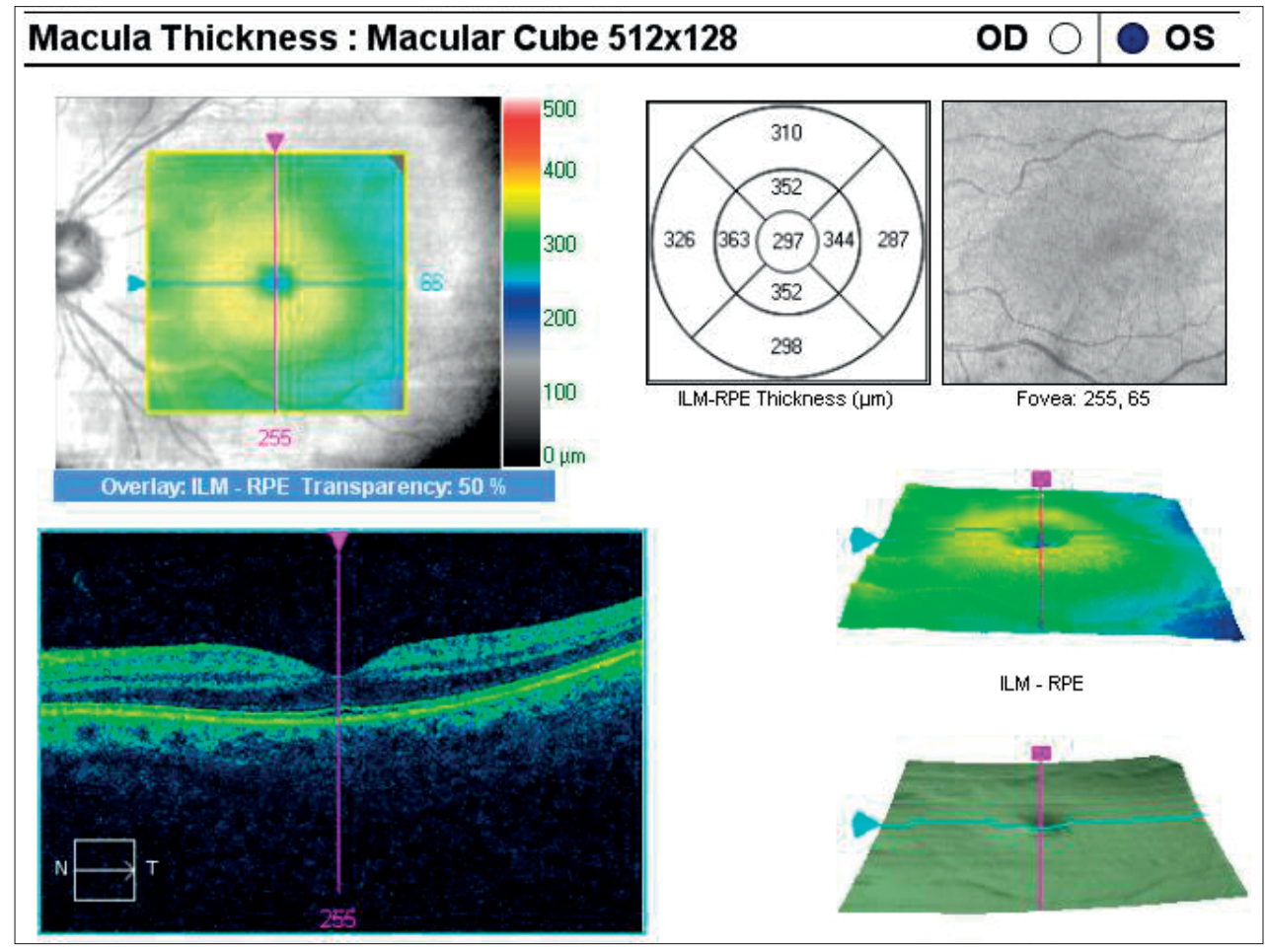

Fig. 6. Resolution of epiretinal membrane.

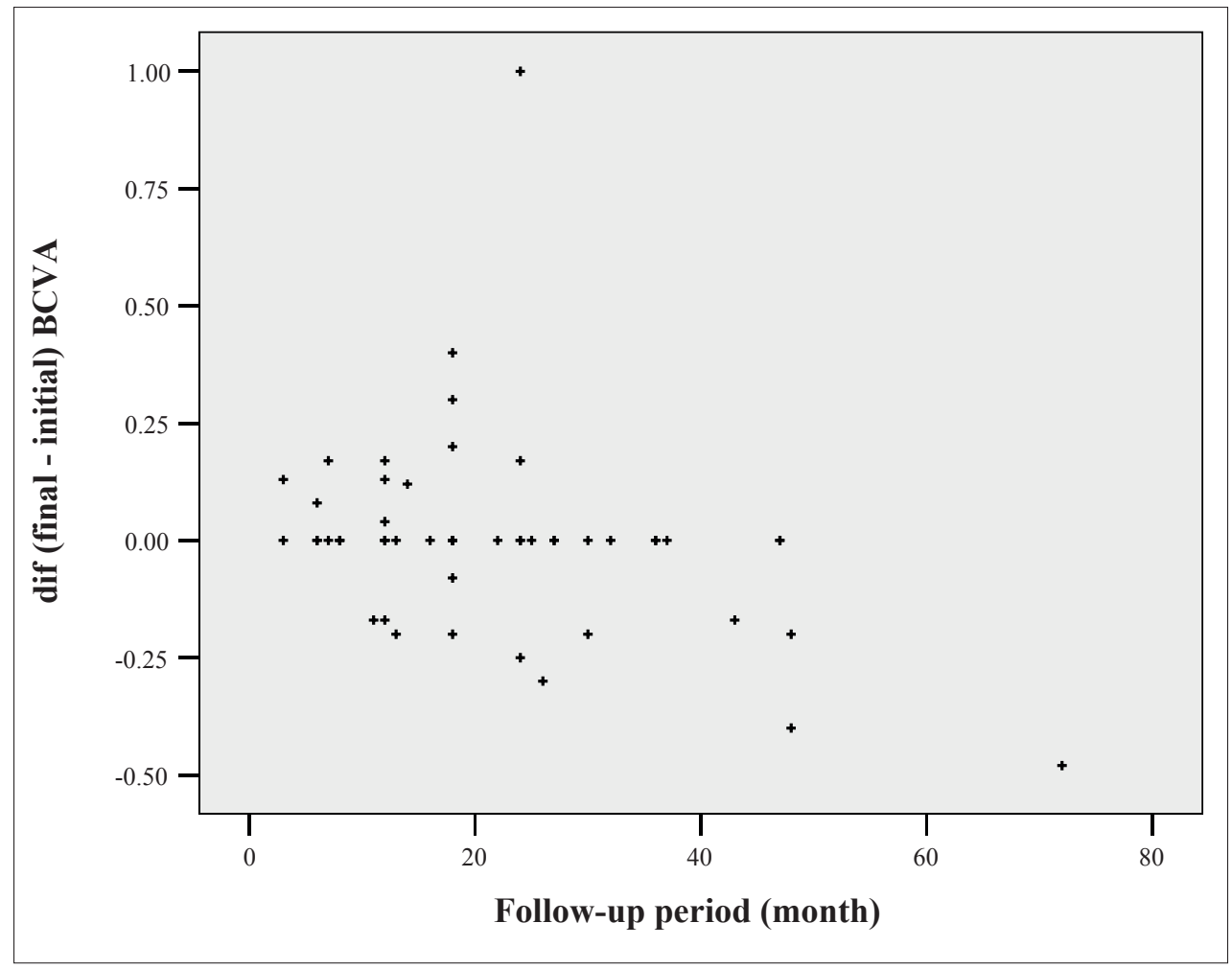

Fig. 7. Dependence of changes in BCVA (difference between the final and initial BCVA) during the follow-up, Spearman $=-0.351, P=0.010$. 


\section{RESULTS}

The age of the patients under investigation ranged from 51 to 85 years (median 72). The average follow-up period was 21.3 months $( \pm 14)$. The initial visual acuity (BCVA) was from 0.8 to -0.10 (LogMAR), mean 0.1 . Approximate Snellen equivalent (20/125 to 20/16, mean 20/25) was not statistically significantly different from the final BCVA from 1.0 to -0.3 , mean 0.1 (LogMAR) (20/200-20/10, mean 20/25) (Table 2). The initial BCVA was significantly correlated with initial CRT, final CRT, final volume and also age. The final BCVA was significantly correlated with all investigated parameters. The initial CRT was $255-819 \mu \mathrm{m}$ (mean, $376.5 \pm 96.9$ ) and the final CRT was $175-600 \mu \mathrm{m}$ (mean, $372.8 \pm 100.7$ ). The input volume of the macula reached the range of $9.4-14.5$ (mean $11.5 \pm 1.0$ ) and the output volume ranged from 9.8 - 15.0 (mean, $11.5 \pm 1.3$ ). Neither parameter measured by OCT showed statistically significant changes. Input and output parameters of the cohort under investigation are shown in Table 1. Nevertheless, the difference between the final and initial BCVA had a statistically significant tendency to decrease during the follow-up (Spearman $=$ -0.351, $P=0.010$, (statistical significance $P \leq 0.05)$ ) (Fig 7). This development of BCVA was not accompanied by corresponding changes in CRT or macular volume.

\section{DISCUSSION}

Idiopathic ERM is a relatively common pathology in the elderly population. ERM is usually asymptomatic and is often diagnosed accidentally. Its surgical treatment is relatively simple and there exist a large number of research papers on surgical results of this diagnosis. On the other hand, there are only a few items of information on the natural course of this disease. From this aspect, the present results are unique and they cannot be practically compared with those in papers by other authors.

In the course of a relatively long follow-up period (on the average 21.3 months) in the present cohort of 53 eyes, no statistically significant anatomical or functional impairment was observed. BCVA remained constant in 29 eyes $(54.7 \%)$, improvement by one or more lines was observed in 12 eyes $(22.6 \%)$, and impairment by one or more lines of the ETDRS optotype was found in 12 eyes $(22.6 \%)$. Only 3 eyes were worse by more than one line (5.6\%). The disease thus seems to be, at least at the beginning, functionally stable.

On the other hand, the photographic examination revealed that ERM is not necessarily of stationary character. Its range and the intensity of reflections may change in time or they may change their localization. (Figs. 1-3). Dystopia of the vessels and their centripetal and centrifugal movement may occur. In the cases of spontaneous improvement in BCVA, a decrease in reflections, or their shift outside the fovea was observed. Anatomical changes were observable also in stable BCVA. Similar results with quantification of tangential retinal movements have already been published ${ }^{8}$. Retinal vessel movements correlated with worsening of BCVA and increased CRT, and were more pronounced in patients with worsening of symptoms.

Initial and final CRT as well as the volume of the macula showed no statistically significant differences. In spite of this, even according to OCT examination, ERM is not stationary in time. The localization of pathology and its seriousness (oedema and its localization) may also change over time. An example is shown in Figs. 4-6, presenting OCT findings corresponding to the retinas in Photographs 1-3. The mean initial CRT was $376.5 \pm 96.9$ and the final CRT $372.8 \pm 100.7 \mu \mathrm{m}$.

Both values corresponded to the mean BCVA 20/25. On the contrary, normal average macular thickness with SD-OCT (Zeiss Cirrus) is $257.6 \pm 19.6 \mu \mathrm{m}$ (ref. ${ }^{9}$ ). BCVA in the initial stage of the disease is not damaged despite a larger thickness of the retina: patients with vision 20/20 had an average CRT of $325 \mu \mathrm{m}$, and in those with the vision 20/25 the average CRT was $369 \mu \mathrm{m}$. At the end of the follow-up period, the CRT of the patients who maintained $\mathrm{BCVA} \geq 20 / 25$ was $318 \mu \mathrm{m}$.

Although in the cohort no statistically significant change was observed in the parameters under study, there is a visible tendency towards a decrease in BCVA during follow-up. However, the tendency towards a decrease in BCVA did not correlate with CRT or the volume of the macula, and therefore we assume that a functional defect precedes the anatomical manifestations of the disease.

\section{CONCLUSION}

BCVA during follow-up tended to decrease: difference of starting BCVA and final BCVA values depending on the time of monitoring is significant. This we attribute to a slow gradual progression of macular changes. But, initial and final BCVA measurements were not substantially different at the end. Thus, in the absence of any clear signs of ERM progression, we can safely postpone the decision whether to perform PPV.

No anatomical progression in ERM within the average follow-up period of 21 months was observed. The indication for surgical treatment is elective and depends on many ocular, extraocular and also psychosocial factors. Functional changes in the tendency towards a decrease in BCVA preceded anatomical changes.

Acknowledgment: This study was supported in part by research project P37/07 (PRVOUK) from the Ministry of Health, Prague, Czech Republic.

Author contributions: All co-authors have read the final manuscript within their respective areas of expertise and participated sufficiently in the study to take responsibility for it and accept its conclusions.

Conflict of interest statement: The authors state that there are no conflicts of interest regarding the publication of this article. 


\section{REFERENCES}

1. Klein R, Klein BE, Wang Q, Moss SE. The epidemiology of epiretinal membranes. Trans Am Ophthalmol Soc 1994;92:403-30.

2. Fraser-Bell S, Guzowski M, Rochtchina E, Wang JJ, Mitchell P. Five-year cumulative incidence and progression of epiretinal membranes: the Blue Mountains Eye Study. Ophthalmology 2003;110(1):34-40.

3. Mitchell P, Smith W, Chey T, Wang JJ, Chang A. Prevalence and as sociations of epiretinal membranes. The Blue Mountains Eye Study, Australia. Ophthalmology 1997;104(6):1033-40.

4. Johnson MW. Epiretinal membrane. In: Yanoff M, Duker J, eds. Ophthalmology. 2nd ed. St Louis: Mosby; 2004.
5. Kampik A. Pathology of epiretinal membrane, idiopathic macular hole, and vitreomacular traction syndrome. Retina 2012;32:194-9.

6. Okamoto F, Sugiura Y, Okamoto Y, Hiraoka T, Oshika T. Time course of changes in aniseikonia and foveal microstructure after vitrectomy for epiretinal membrane. Ophthalmology 2014;121(11):2255-60.

7. Regillo C. Epiretinal membranes. Retina and Vitreous: Basic and clinical science course, San Francisco: American Academy of Ophthalmology 2011; section 12.

8. Kofod M, la Cour M. Quantification of retinal tangential movement in epiretinal membranes. Ophthalmology 2012;119(9):1886-91.

9. Kakinoki M, Sawada O, Sawada T, Kawamura H, Ohji M. Comparison of macular thickness between Cirrus HD-OCT and Stratus OCT. Ophthalmic Surg Lasers Imaging 2009;40(2):135-40. 